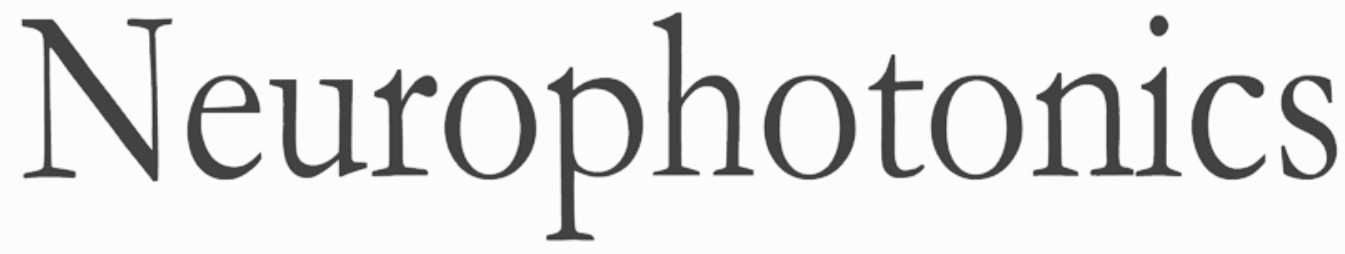

\title{
Emerging research areas in need of neurophotonics: report from the 2014 Aarhus Capillary Transit Time Heterogeneity (CTH) meeting
}

Leif Østergaard

Sava Sakadžić 


\title{
Emerging research areas in need of neurophotonics: report from the 2014 Aarhus Capillary Transit Time Heterogeneity (CTH) meeting
}

\author{
Leif Østergaard, MD, PhD, Professor \\ Aarhus University \\ Center for Functionally Integrative Neuroscience \\ Department of Neuroradiology \\ Aarhus University Hospital \\ Nørrebrogade 44, Building 10G \\ DK-8000 Aarhus C, Denmark \\ E-mail: leif@cfin.au.dk
}

\author{
Sava Sakadžić, PhD, Instructor in Radiology \\ Optics Division \\ Martinos Center for Biomedical Imaging \\ Massachusetts General Hospital \\ Harvard Medical School \\ 149 13th Street, Room 2301 \\ Charlestown, Massachusetts 02129 \\ E-mail: sava.sakadzic@mgh.harvard.edu
}

Each December since 2012, scientists from a range of disciplines have convened in Aarhus, Denmark, to piece together a better understanding of the brain's microcirculation. Members of the neurophotonics community, the microcirculation community, and scientists interested in measuring the brain's blood flow and metabolism have met to discuss how blood flow and oxygen transport in the microcirculation can be imaged and modeled, and how it supports brain function in health and disease. From these workshops, it is evident that the rapid advances in neurophotonics are playing an increasingly important role in advancing our understanding of how cerebral metabolic needs are met by cerebral blood flow. Here, we briefly summarize the most recent workshop in hopes of inspiring others to consider applying their tools to this important problem.

The background for these meetings is the realization that we need a better understanding of the brain's microcirculation in order to understand devastating brain disorders such as dementia, the severe disabilities that develop after stroke and head trauma, psychiatric disorders, and possibly our difficulties in treating brain cancers. Also, our efforts to map human brain function relies on methods that detect changes in cerebral blood flow and oxygen utilization, but the exact relation between such changes and the underlying neuronal processes remains unclear.

Until recently, brain function has mainly been interrogated by "macroscopic" imaging techniques that are sensitive to cerebral blood flow (CBF) and the brain's utilization of oxygen and glucose. These techniques include functional magnetic resonance imaging (fMRI) to detect changes in CBF or blood-oxygenation in relation to changes in brain activity, and positron emission tomography (PET) to quantify the uptake of radiolabeled oxygen or glucose analogs. These methods are crucial not only in the study of brain function but also in the identification of patients where vascular disorders limit the oxygen supply to certain brain regions. In particular, the

(C) 2016 Society of Photo-Optical Instrumentation Engineers (SPIE) localization of brain function by "macroscopic" parameters such as CBF is based on the common assumption that CBF changes according to changes in local brain activity - socalled neurovascular coupling. At the same time, text-book physiology predicts a one-to-one relation between CBF and oxygen availability in brain tissue. Combined, this permits us to rule out vascular causes of symptoms in patients provided their CBF images show normal values.

The use of CBF sensitive techniques to characterize brain activity and tissue oxygenation may, however, be highly misleading. While adequate macroscopic CBF is clearly a prerequisite for proper brain oxygenation, an adequate flow distribution across the capillary bed is crucial for tissue to be able to extract oxygen from blood. The term capillary transit time heterogeneity $(\mathrm{CTH})$ refers to this aspect of oxygen transport and to the fact that both CBF and CTH must be known in order to infer the brain's access to oxygen and nutrients such as glucose. ${ }^{1}$ In order to improve our understanding of these complex phenomena, we need in vivo imaging tools that can measure cerebral blood flow characteristics at the microscopic level. These methods should preferably image a whole microvascular network over a millimeter cortical area and with significant cortical depth penetration, and with a temporal resolution comparable to the timescale of the CBF response to brain activation $(\sim 1 \mathrm{~s})$.

Optical microscopy technologies are ideally suited for this task. Two-photon microscopy is already routinely used to track RBC passage in a few selected vessels but further improvements are needed to enable faster sampling of the larger microvascular populations. The last few years have witnessed a rapid expansion of CBF imaging based on optical coherence tomography (OCT), which can rapidly measure both absolute CBF in penetrating arterioles and venules and RBC flux in capillaries. At the last CTH meeting in December 2014, Jonghwan Lee (MGH / HMS, Charlestown) presented recent advances in the use of OCT to quantify capillary flow velocities over a volume approaching a cubic millimeter. ${ }^{2}$ Recent progress with photoacoustic blood flow measurements indicate that photoacoustics may soon realize the 
potential of mapping microvascular blood flow characteristics over even larger volumes of tissue.

The study of the microcirculation is a mature field within physiology and vascular biology, and the CTH meetings have benefitted from the willingness of several luminaries within this field to help organize the meetings and to share their knowledge from decades of research and modeling. Axel Pries (Charité, Berlin), a pioneer within the study of relations between capillary morphology, topology, and flow dynamics, co-organizes the $\mathrm{CTH}$ meetings, and at the last meeting, two former presidents of the Microcirculatory Society, Roland N. Pittman (VCU Medical Center, Richmond, VA), and Timothy W. Secomb (University of Arizona, Tucson), gave inspiring lectures on the regulation of oxygen transport in tissue and on advanced models of oxygen transport in tissue..$^{3,4}$ Models of capillary flow distributions by Pries and Secomb have shown that signaling within the capillary bed is crucial to ensure that the blood simply does not follow the shortest paths to the venous system but instead follows longer capillary paths as well to ensure that the tissue is adequately supplied with oxygen. ${ }^{5}$ They pioneered the idea that "functional shunts" may form in abnormal tumor vasculature and cause the hypoxia that makes cancers more aggressive and resistant to treatment. ${ }^{6}$ At the meeting, Anne Tietze (CFIN, Aarhus University) presented the first evidence of this phenomenon in data from human brain tumor patients. ${ }^{7}$

The distribution of blood-cells across the capillary bed is determined by a range of biophysical properties of both the capillary network itself and of the flowing blood. Janine Berkholz (Charité, Berlin) and Hans Vink (Maastricht University, Holland) described how the inner lining of the capillary lumen, the glycocalyx, controls capillary flows and may contribute to several cardiovascular disorders. ${ }^{8,9}$ The modeling of capillary blood flow and oxygen transport in tissue has been a central theme in past CTH meetings, and at the last meeting, Patrick Jenny (ETH, Zürich) demonstrated how computational fluid dynamics and models of oxygen advection in tissue may help our understanding of tissue oxygenation. ${ }^{10}$

Computational models and experimental observations of oxygen transport in tissue are mutually dependent. To make further progress on both fronts, it is critical not only to measure the detailed CBF distribution but also to map oxygen distribution in both the microvasculature and tissue. The challenge of mapping intravascular oxygenation now seems to be within reach for several optical imaging technologies, including TPM imaging of oxygen partial pressure (PO2), photoacoustic microscopy imaging of hemoglobin oxygen saturation (SO2), and visible-light OCT imaging of SO2. In addition, TPM imaging of $\mathrm{PO} 2$ enables high-resolution mapping of cerebral tissue $\mathrm{PO} 2$, but further improvements are needed to increase the measurement speed. Sava Sakadzic (MGH/HMS, Charlestown) presented a combination of TPM imaging of $\mathrm{PO} 2$ and OCT imaging of CBF in mouse brain with numerical modeling and showed that oxygen exchange has a large arteriolar component in anesthetized resting brain, while capillary oxygen extraction dominates during metabolic and CBF perturbations. ${ }^{11}$

Accurate imaging of the microvascular morphology and its segmentation represents another challenge. TPM angiography and associated segmentation algorithms are presently the most advanced among the optical imaging technologies. However, accurate microvascular segmentation still requires significant manual labor and experimental issues with penetration depth, aberration-induced artifacts, and spatial and temporal resolution are negatively impacting the current ability of optical imaging methods to rapidly acquire sufficiently large and accurate angiograms in vivo. In addition, intensified efforts on awake animal imaging are needed to provide physiologically more relevant structural and functional data.

The coupling between brain metabolism and blood flow has been a central topic for previous CTH meetings. This year, Richard Buxton (USCD, La Jolla), the leading authority on neurovascular coupling and models of blood oxygenation relevant to $\mathrm{fMRl}$, discussed recent advances in our understanding of the factors that govern the regulation of blood flow, emphasizing the importance of maintaining tissue oxygen tension to drive central metabolic processes. ${ }^{12}$ Jozien Goense (Glasgow University, UK), highlighted the need for optical observations in the study of these mechanisms: Using ultra-high resolution $\mathrm{FMRI}$, she was able to demonstrate that "macroscopic" fMRI data in fact represent "lumped" signals from widely different tissue responses within individual cortical layers in the brain. ${ }^{13}$

\section{Conclusion}

With this report, we wish to highlight an emerging, fertile research area in which the development of innovative optical methods plays a crucial role in the multidisciplinary effort to understand brain function and brain disorders. Novel methods to image microvascular flow dynamics, oxygen transport, and cell function are likely to improve not only our understanding of how the metabolic needs of normal brain function are met but also to help us understand the origin of a range of brain disorders. We hope that the summary provided here, with details in the provided citations, will motivate those developing novel neurophotonic tools to apply them to these intriguing problems.

\section{References}

1. S. N. Jespersen and L. Østergaard, "The roles of cerebral blood flow, capillary transit time heterogeneity and oxygen tension in brain oxygenation and metabolism," J. Cereb. Blood Flow Metab. 32, 264 277 (2012).

2. J. Lee et al., "Multiple-capillary measurement of RBC speed, flux, and density with optical coherence tomography," J. Cereb. Blood Flow Metab. 33, 1707-1710 (2013).

3. B. Reglin, T. W. Secomb, and A. R. Pries, "Structural adaptation of microvessel diameters in response to metabolic stimuli: where are the oxygen sensors?" Am. J. Physiol. Heart Circ. Physiol. 297, H2206-H22019 (2009).

4. R. N. Pittman, "Oxygen transport in the microcirculation and its regulation," Microcirculation 20, 117-137 (2013).

5. A. R. Pries and T. W. Secomb, "Making microvascular networks work: angiogenesis, remodeling, and pruning," Physiology (Bethesda) 29, 446-455 (2014)

6. A. R. Pries et al., "The shunt problem: control of functional shunting in normal and tumour vasculature," Nat. Rev. Cancer 10, 587-593 (2010).

7. A. Tietze et al., "Perfusion MRI derived indices of microvascular shunting and flow control correlate with tumor grade," PLoS One 10(4), e0123044, (2015). 
8. H. Vink and B. R. Duling, "Identification of distinct luminal domains for macromolecules, erythrocytes, and leukocytes within mammalian capillaries," Circ. Res. 79, 581-589 (1996).

9. A. R. Pries and W. M. Kuebler, "Normal endothelium," Handb. Exp. Pharmacol. 176(Pt. 1), 1-40 (2006).

10. A. Lucker, B. Weber, and P. Jenny, "A dynamic model of oxygen transport from capillaries to tissue with moving red blood cells," Am. J. Physiol. Heart Circ. Physiol. 308, H206-H216 (2015).
11. S. Sakadzic et al., "Large arteriolar component of oxygen delivery implies a safe margin of oxygen supply to cerebral tissue," Nat. Commun. 5, 5734 (2014).

12. R. B. Buxton, "Dynamic models of BOLD contrast," NeuroImage 62, 953-961 (2012).

13. J. Goense, H. Merkle, and N. K. Logothetis, "High-resolution fMRI reveals laminar differences in neurovascular coupling between positive and negative BOLD responses," Neuron 76, 629-639 (2012). 